\title{
Influence of glutathione S-transferase polymorphisms on type- 2 diabetes mellitus risk
}

\author{
M.A. Amer ${ }^{1}$, M.H. Ghattas ${ }^{2}$, D.M. Abo-EIMatty ${ }^{3}$ and S.H. Abou-El-Ela ${ }^{3}$ \\ ${ }^{1}$ Department of Pharmacology, Faculty of Pharmacy, \\ Sinai University, El-Arish, Egypt \\ ${ }^{2}$ Department of Medical Biochemistry, Faculty of Medicine, \\ Suez Canal University, Ismailia, Egypt \\ ${ }^{3}$ Department of Biochemistry, Faculty of Pharmacy, \\ Suez Canal University, Ismailia, Egypt \\ Corresponding author: M.A. Amer \\ E-mail:ph.maa@su.edu.eg
}

Genet. Mol. Res. 10 (4): 3722-3730 (2011)

Received May 18, 2011

Accepted September 28, 2011

Published October 31, 2011

DOI http://dx.doi.org/10.4238/2011.October.31.14

\begin{abstract}
Glutathione S-transferase (GST) protects cells against oxidative stress. We evaluated the effect of genetic polymorphisms of the GST gene family on the risk of developing type-2 diabetes mellitus and on glycemic control. We also investigated the effects of smoking combined with these polymorphisms on type- 2 diabetes mellitus risk. We enrolled 100 type- 2 diabetes mellitus patients and 100 healthy controls matched for age, gender and origin, from the Sinai area of Egypt. Fasting serum glucose, $\mathrm{HbA}_{1 \mathrm{c}}$ and lipid profiles were determined. Two polymorphisms were identified by multiplex PCR within the GST genes: GSTM1 and GSTT1. The proportion of the GSTT1- and GSTM1-null genotypes was significantly greater in diabetic patients when compared to controls. Patients carrying both null polymorphisms had a 3.17 -fold increased risk of having type-2 diabetes mellitus compared to those with normal genotypes of these two genes $(\mathrm{P}=0.009)$. Additionally, patients with the GSTT1-null genotype had higher levels of triglycerides and very low-density lipoprotein cholesterol compared to those with the
\end{abstract}


GSTT1-present genotype. On the other hand, patients with the GSTM1null genotype had significantly higher levels of $\mathrm{HbA}_{1 \mathrm{c}}$ and significantly higher diastolic blood pressure compared to those with the GSTM1present genotype. The interaction between these genotypes and smoking status was not significant. These results give evidence that the GSTT1and GSTM1-null genotypes, alone or combined, are associated with increased risk of type-2 diabetes mellitus, regardless of smoking status. Only the GSTM1-null genotype had an effect on glycemic control.

Key words: Glutathione S-transferase; Polymorphism; Multiplex PCR; Type-2 diabetes mellitus

\section{INTRODUCTION}

Diabetes has become an epidemic and remains a major public health issue. In 2010, it was estimated that 4.787 million Egyptians (10.4\% of the Egyptian population) had diabetes and that diabetes will increase to 8.615 million Egyptians by the year 2030 (Shaw et al., 2010).

Oxidative stress is defined in general as excess formation and/or insufficient removal of highly reactive molecules such as reactive oxygen species (ROS) (Maritim et al., 2003). ROS contribute to the pathogenesis of various chronic diseases, including diabetes mellitus (Baynes, 1991). Pancreatic $\beta$-cells have emerged as a putative target of oxidative stress-induced tissue damage, and this seems to explain in part the progressive deterioration of $\beta$-cell function in type 2 diabetes mellitus (T2DM) (Gorogawa et al., 2002).

Endogenous antioxidant enzymes exist to inactivate ROS. One of these enzymes is glutathione S-transferase (GST), which carries out a wide range of functions in cells: removal of ROS and regeneration of S-thiolated protein (as consequences of oxidative stress) and conjugation with endogenous ligands, as well as reactions in metabolic pathways not associated with detoxification (Sheehan et al., 2001). As a phase II biotransformation enzyme, GST catalyzes the conjugation of reduced glutathione (GSH) to electrophilic centers on a wide range of substrates (Douglas, 1987).

In humans, glutathione-S-transferases supergene family (GST; EC 2.5.1.18) have been assigned to at least eight separate classes designated alpha, mu, kappa, omega, pi, sigma, theta and zeta, which are encoded by the GSTA, GSTM, GSTK, GSTO, GSTP, GSTS, GSTT, and GSTZ genes, respectively (Hayes and Strange, 2000).

Several GST polymorphisms have been associated with an increased or decreased susceptibility to several diseases. Deletion polymorphisms in the GSTM1 and GSTT1 genes, as important members of the GST family, result in complete lack of GSTM1 and GSTT1 proteins (Pemble et al., 1994). Recently, the GSTT1 null genotype or both the GSTT1 and GSTM1 null genotypes have been shown to be a genetic risk factor, namely interacting with current-smoking status, for the development of type 2 diabetes and its cardiovascular complications (Doney et al., 2005; Hori et al., 2007).

The present work aimed to study the influence of the genetic polymorphisms of the GST gene on clinical parameters of T2DM in order to add to the existing knowledge of the genetic basis of the disease in the Egyptian population. Additionally, genotyping may help in the early diagnosis and management of T2DM. 


\section{MATERIAL AND METHODS}

\section{Subjects}

The study groups included 100 patients with type 2 DM (45 men, 55 women) who were diagnosed according to the American Diabetes Association criteria (American Diabetes Association, 2007) and 100 healthy controls (53 men, 47 women). All patients and controls were unrelated individuals from the Egyptian Sinai area. Fasting blood specimens were collected. Hypertension was defined as a systolic blood pressure (BP) $\geq 140 \mathrm{mmHg}$, a diastolic $\mathrm{BP} \geq 90 \mathrm{mmHg}$, or both. Current smoker was defined as a subject who continued to smoke cigarettes regularly. The present study was conducted according to the principles of the Declaration of Helsinki, and all patients provided written informed consent, following a protocol approved by the Suez Canal University Research Ethics Committee. Table 1 summarizes the clinical features of patients and controls.

\begin{tabular}{|c|c|c|c|}
\hline & Control $(\mathrm{N}=100)$ & T2DM $(\mathrm{N}=100)$ & $P$ value \\
\hline Age (years) & $48.31 \pm 7.8$ & $48.77 \pm 7.01$ & 0.66 \\
\hline Gender (female/male) & $47 / 53$ & $55 / 45$ & 0.26 \\
\hline Fasting blood glucose (mg/dL) & $96.16 \pm 9.34$ & $206.89 \pm 87.5$ & $0.0001^{*}$ \\
\hline $\mathrm{HbA}$ (\%) & $4.99 \pm 0.72$ & $9.63 \pm 1.8$ & $0.0001 *$ \\
\hline BMI & $28.55 \pm 4.01$ & $29.92 \pm 3.85$ & $0.015^{*}$ \\
\hline Total cholesterol (mg/dL) & $204.75 \pm 73.84$ & $217.93 \pm 44.96$ & 0.13 \\
\hline $\mathrm{TG}(\mathrm{mg} / \mathrm{dL})$ & $149.65 \pm 71.45$ & $174.21 \pm 88.76$ & $0.03 *$ \\
\hline HDL-C (mg/dL) & $46.72 \pm 10.53$ & $45.39 \pm 10.39$ & 0.37 \\
\hline VLDL-C (mg/dL) & $31.46 \pm 15.3$ & $35.23 \pm 17.84$ & 0.11 \\
\hline LDL-C $(\mathrm{mg} / \mathrm{dL})$ & $126.57 \pm 74.85$ & $137.31 \pm 47.47$ & 0.23 \\
\hline Current smoking $(+/-)$ & $19 / 81$ & $28 / 72$ & 0.13 \\
\hline Systolic blood pressure (mmHg) & $121.4 \pm 15.19$ & $138.3 \pm 28.69$ & $0.0001 *$ \\
\hline Diastolic blood pressure (mmHg) & $75.35 \pm 7.86$ & $82.85 \pm 12.42$ & $0.0001 *$ \\
\hline
\end{tabular}

Comparisons were performed by the Student $t$-test and the chi-square test. Data are reported as means $\pm \mathrm{SD}$. * Significant differences between groups $(\mathrm{P}<0.05)$.

\section{Genomic DNA extraction and genotyping}

Genomic DNA was isolated from $100 \mu \mathrm{L}$ whole blood collected in EDTA anticoagulated tubes using the Wizard genomic DNA purification kit (Promega, Madison, USA). GSTM1 and GSTT1-null alleles were analyzed using a multiplex PCR reaction with beta globin as an internal control. PCR amplifications were performed in a total volume of 25 $\mu \mathrm{L}$ containing $50 \mathrm{ng}$ genomic DNA, 1 X PCR buffer, $3 \mathrm{mM} \mathrm{MgCl}, 400 \mu \mathrm{M}$ dNTPs, $1.5 \mathrm{U}$ Taq polymerase (Promega) and $4 \mu \mathrm{M}$ of each primer as follows: GSTM1 forward primer 5'-GAACTCCCTGAAAAGCTAAAGC-3', reverse primer 5'-GTTGGGCTCAAATATACGG TGG-3', GSTT1 forward primer 5'-TTCCTTACTGGTCCTCACATCTC-3', reverse primer 5'-TCACCGGATCATGGCCAGCA-3' and $\beta$-globin forward primer 5'-CAACTTCATCCAC GTTCACC-3', reverse primer 5'-GAAGAGCCAAGGACAGGTAC-3'. Thermal cycling conditions were as follows: an initial denaturation step at $95^{\circ} \mathrm{C}$ for $5 \mathrm{~min}, 35$ cycles at $94^{\circ} \mathrm{C}$ for $1 \mathrm{~min}$, $60^{\circ} \mathrm{C}$ for $1 \mathrm{~min}$ and $72^{\circ} \mathrm{C}$ for $1 \mathrm{~min}$, and a final extension step at $72^{\circ} \mathrm{C}$ for $5 \mathrm{~min}$. The amplification products were size separated on $2 \%$ agarose gels and visualized by ethidium bromide staining. GSTM1 \& GSTT1 genotypes were determined by the presence and absence (null) of bands 
of $210 \mathrm{bp}$ and $480 \mathrm{bp}$, respectively, with an internal control of $260 \mathrm{bp}$. Using this genotyping assay of GSTM1 and GSTT1, the null genotypes can be clearly categorized, but the heterozygote and homozygote positive genotypes could not be differentiated (Shaikh et al., 2010) (Figure 1).

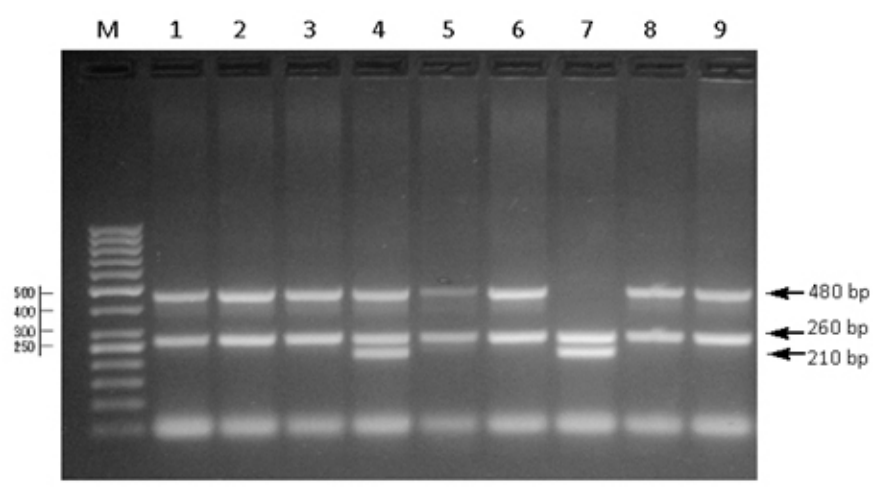

Figure 1. PCR products analyzed on 2\% agarose gel. The presence or absence (null) of GSTM1 and GSTT1 was detected by the presence or absence of a band at $480 \mathrm{bp}$ (corresponding to GSTT1) and a band at $210 \mathrm{bp}$ (corresponding to GSTM1). $\beta$-Globin is considered an internal control (260 bp). 50 bp ladder Lane 1; GSTM1 and GSTT1 wild type Lane 4; GSTM1 null Lanes 1-3, 5, 6, 8, 9; GSTT1 null genotype Lane 7.

\section{Statistical analysis}

Statistical analyses were performed with the SPSS ver. 17 software package. The relationship between GSTT1 and GSTM1 genotypes and risk of T2DM was assessed by the means of the odds ratio (OR) with $95 \%$ confidence limits calculated by logistic regression. GSTT1 and GSTM1 genotypes were classified as either null (homozygous deletion) or non-deleted. Data are expressed as mean \pm S.D. Frequencies of categorical variables were compared by the $\chi^{2}$ test. Associations of genotypes with biochemical findings were evaluated by the two-way the Student $t$-test. Multiple regression analysis was used to adjust for confounding factors. $\mathrm{P}<$ 0.05 was taken as indicative of significant differences.

\section{RESULTS}

\section{Clinical variables}

One hundred subjects (45 male and 55 female) were diagnosed as having type 2 DM. Comparisons were made between diabetic subjects and their age- and gender-matched control subjects (53 male and 47 female). The relevant characteristics of the subjects studied are shown in Table 1. Among all clinical characteristics of diabetics, only serum fasting glucose, $\mathrm{HbA}_{1 \mathrm{c}}$, BMI, TG, systolic blood pressure and diastolic blood pressure levels showed a significant association when compared to controls (Table 1).

\section{Distribution of the GST genotypes and combination of the genotypes in the subjects}

In diabetic patients, the frequencies of GSTT1-null and GSTM1-null genotypes were 
35 and $62 \%$, respectively. Meanwhile, in the control samples, the frequencies of GSTT1-null and GSTM1- null genotypes were 21 and 47\%, respectively. The proportion of the GSTT1null and GSTM1-null genotypes increased in diabetic patients when compared to controls ( $\mathrm{P}$ $=0.027$ and $\mathrm{P}=0.033$, respectively) (Table 2 ).

\begin{tabular}{|c|c|c|c|c|}
\hline & Control $(\mathrm{N}=100)$ & $\mathrm{T} 2 \mathrm{DM}(\mathrm{N}=100)$ & OR $(95 \% \mathrm{CI})$ & $P$ value \\
\hline \multicolumn{5}{|l|}{ GSTT1 } \\
\hline Null (-) & 21 & 35 & 1 & \\
\hline Present $(+)$ & 79 & 65 & $0.49(0.26-0.93)$ & $0.027^{*}$ \\
\hline \multicolumn{5}{|l|}{ GSTM1 } \\
\hline Null (-) & 47 & 62 & 1 & \\
\hline Present $(+)$ & 53 & 38 & $0.54(0.31-0.95)$ & $0.033^{*}$ \\
\hline \multicolumn{5}{|l|}{ GSTT1 and GSTM1 } \\
\hline Both present $(+/+)$ & 44 & 22 & 1 & \\
\hline Either null $(+/-)+(-/+)$ & 44 & 59 & $2.68(1.4-5.1)$ & $0.002 *$ \\
\hline Both null $(-/-)$ & 12 & 19 & $3.17(1.31-7.68)$ & $0.009^{*}$ \\
\hline
\end{tabular}

Comparisons were performed by the chi-square test. * Significant differences between groups $(\mathrm{P}<0.05)$.

The incidence of both present genotypes occurring together was associated with decreased risk of having type 2 diabetes relative to the either or both null genotypes. The combination of the two high-risk genotypes either GSTT1-null or GSTM1-null, was associated with a 2.68 -fold increased risk relative to the both present genotypes $(\mathrm{P}=0.002)$ and the incidence of both null polymorphisms occurring together was associated with a 3.17 -fold increased risk of having type 2 diabetes relative to the both present genotypes $(\mathrm{P}=0.009)$ (Table 2).

\section{Genetic polymorphism influence on clinical parameters}

We further investigated the clinical parameters accompanying high risk genotypes (GSTT1-nu 11 or GSTM1-null) compared to non-risk genotypes (GSTT1-present and GSTM1-present genotypes) in diabetic patients. In cases of GST1-null genotype, there were higher levels of triglycerides and VLDL-C compared to the GSTT1-present genotype ( $\mathrm{P}=$ 0.042 and $\mathrm{P}=0.037$, respectively) (Table 3 ).

\section{Table 3. The relationship between GSTT1 genotype and different clinical parameters in diabetic patients.}

\begin{tabular}{lccc}
\hline & Null & Present & P value \\
\hline $\mathrm{N}$ (female/male) & $35(20 / 15)$ & $65(35 / 30)$ & 0.75 \\
Fasting blood glucose (mg/dL) & $228.77 \pm 112.74$ & $195.11 \pm 68.54$ & 0.07 \\
$\mathrm{HbA}_{1 \mathrm{c}}$ (\%) & $9.42 \pm 1.94$ & $9.75 \pm 1.74$ & 0.39 \\
BMI & $29.58 \pm 4$ & $30.10 \pm 3.78$ & 0.52 \\
Total cholesterol (mg/dL) & $214.8 \pm 48.37$ & $219.62 \pm 43.32$ & 0.61 \\
TG (mg/dL) & $198.66 \pm 102.82$ & $161.04 \pm 77.89$ & 0.75 \\
VLDL-C (mg/dL) & $40.29 \pm 20.67$ & $32.5 \pm 15.62$ & $0.037 *$ \\
LDL-C (mg/dL) & $129.57 \pm 25.52$ & $141.48 \pm 44.94$ & 0.15 \\
Current smoking (+/-) & $9 / 26$ & $19 / 46$ & 0.71 \\
Systolic blood pressure (mmHg) & $136.57 \pm 25.5$ & $139.23 \pm 30.4$ & 0.66 \\
Diastolic blood pressure (mmHg) & $81.86 \pm 10.3$ & $83.38 \pm 13.47$ & 0.56 \\
\hline
\end{tabular}

Comparisons were performed by the Student $t$-test and the chi-square test. Data are reported as means $\pm \mathrm{SD}$. *Significant differences between groups $(\mathrm{P}<0.05)$. 
In cases of the GSTM1-null genotype, there were higher levels of $\mathrm{HbA}_{1 \mathrm{c}}$ and diastolic blood pressure compared to GSTM1-present genotype ( $\mathrm{P}=0.027$ and $\mathrm{P}=0.02$, respectively) (Table 4). On the other hand, the frequency of the GSTT1 and GSTM1 genotypes did not differ between the diabetes subjects and the non-diabetic ones, and the interactive effect between the genotypes and smoking status was not significant. The GSTT1-null and GSTM1-null genotypes were an independent risk factor for type 2 diabetes, regardless of smoking status.

Table 4. The relationship between GSTM1 genotype and different clinical parameters in diabetic patients.

\begin{tabular}{lccc}
\hline & Null & Present & P value \\
\hline N (female/male) & $62(38 / 24)$ & $38(17 / 21)$ & 0.11 \\
Fasting blood glucose (mg/dL) & $211.23 \pm 83.64$ & $199.82 \pm 94.27$ & 0.53 \\
HbA $(\%)$ & $9.95 \pm 1.65$ & $9.13 \pm 1.95$ & $0.027 *$ \\
BMI & $30.18 \pm 4$ & $29.5 \pm 3.46$ & 0.39 \\
Total cholesterol (mg/dL) & $223.55 \pm 42.37$ & $208.76 \pm 48.07$ & 0.11 \\
TG $(\mathrm{mg} / \mathrm{dL})$ & $179.34 \pm 89.82$ & $165.84 \pm 87.54$ & 0.46 \\
HDL-C (mg/dL) & $46.19 \pm 10.3$ & $44.08 \pm 10.3$ & 0.32 \\
VLDL-C (mg/dL) & $36.27 \pm 18.08$ & $33.52 \pm 17.56$ & 0.46 \\
LDL-C (mg/dL) & $141.08 \pm 46.24$ & $131.16 \pm 49.41$ & 0.31 \\
Current smoking (+/-) & $18 / 44$ & $10 / 28$ & 0.77 \\
Systolic blood pressure (mmHg) & $142.66 \pm 30.44$ & $131.18 \pm 24.29$ & 0.05 \\
Diastolic blood pressure (mmHg) & $85.1 \pm 12.94$ & $79.21 \pm 10.69$ & $0.02 *$ \\
\hline
\end{tabular}

Comparisons were performed by the Student $t$-test and the chi-square test. Data are reported as means $\pm \mathrm{SD}$. *Significant difference between groups $(\mathrm{P}<0.05)$.

\section{DISCUSSION}

Diabetes mellitus is one of the most common chronic diseases in nearly all countries; the number of people with diabetes is increasing due to population growth, aging, urbanization, and increasing prevalence of obesity and reduced physical activity (Wild et al., 2004). It is estimated that Egypt will be listed in the top 10 countries with the highest numbers of people with diabetes in 2030, reflecting anticipated changes in the population size and structure in Egypt (Wild et al., 2004).

Oxidative stress plays a major role in the pathogenesis of T2DM (Stephens et al., 2009). $\beta$-cells are particularly sensitive to ROS because they are low in antioxidant factors such as glutathione peroxidase, catalase and SOD. Therefore, increased oxidative stress may not only result from hyperglycemia associated with diabetes, but may also have an important causal role in $\beta$-cell failure and the development of insulin resistance and T2DM (Stephens et al., 2009).

Glutathione S-transferase (GST) modulates the effects of various cytotoxic and genotoxic agents. GST, along with other antioxidant enzymes, such as Se-dependent GPx1, provides the cell with protection against a range of harmful electrophiles produced during oxidative damage to membranes (Hayes and McLellan, 1999).

GSTs constitute one of the major components of the phase II drug metabolizing enzyme and antioxidant systems (Wang et al., 2006). Therefore, there is an increasing interest in the role that polymorphisms in phase I and phase II detoxification enzymes may play in the etiology and progression of diseases. Polymorphisms reducing or eliminating these enzyme detoxification activities could increase a person's susceptibility to diseases including T2DM. We thus determined the polymorphism frequency for each of these enzymes in our study populations and looked for relationships between them and the clinical parameters in type 2 diabetics. 
There are many studies dealing with GST polymorphism in various diseases, but only a few studies have addressed the role of GST polymorphisms in diabetes and in glycemic control. In the current study, we attempted to move beyond single gene polymorphism to two-gene polymorphisms that may help predict the susceptibility to the incidence of T2DM and their effect on glycemic control in the Egyptian population in the Sinai area.

The deletion polymorphisms of GSTT1 and GSTM1, which are associated with abolished enzyme activity (London et al., 2000), have been associated with type 2 diabetes mellitus when compared to control subjects (Hori et al., 2007; Wang et al., 2006).

The results presented herein showed significantly higher frequencies of GSTM1 and GSTT1 polymorphisms ( $\mathrm{P}=0.033$ and $\mathrm{P}=0.027$ respectively) in $\mathrm{T} 2 \mathrm{DM}$ patients compared to healthy controls (Table 2). The control group had a polymorphic frequency of 47 and $21 \%$, respectively. This is close to the respective frequencies reported for Spanish (49.7 and 20.5\%; Garte et al., 2001), Turkish (51.9 and 17.3\%; Ada et al., 2004), Italian (46.9 and 19\%; D'Alò et al., 2004) and Caucasian (48.8 and 19.9\%; Gsur et al., 2001) control populations. These finding may be explained by the fact that Egyptians belong to the Mediterranean race.

Wang and colleagues (2006) performed a study on Chinese type 2 diabetic patients investigating the polymorphism of GSTT1. They found that the GSTT1-null genotype was observed in $61 \%$ of patients versus $51 \%$ of controls. Their study suggests that the GSTT1-null genotype contributes to the development of type 2 diabetes mellitus. Our study showed a significant difference in the frequencies of the GSTT1-null mutations between the patients and the control group (35vs 21\%, respectively).

The GSTT1-null genotype conferred a statistically significant 0.49 -fold increased risk of T2DM relative to the present genotype of the GSTT1 gene, that is, the individuals are not capable of detoxifying the products of oxidative stress.

On the other hand, we found $62 \%$ homozygosis for the GSTM1 deletion in diabetics and $21 \%$ in the control subjects $(\mathrm{P}=0.03)$. This frequency was in accordance with Bid and colleagues (2010). The presence of the GSTM1-null genotype seems to increase the risk of having T2DM by 0.54 -fold.

Moreover, we found significant differences between the GSTT1-present and GSTM1-present and either or both null genotypes of diabetics; we found significant differences $(\mathrm{P}=0.002$ and $\mathrm{P}=0.009$ respectively) when compared to the control subjects. These results support the notion that GSTT1 and GSTM1 cooperatively play a protective role against the development of type 2 diabetes mellitus.

We also attempted to evaluate the association of GSTM1 (present, null) and GSTT1 (present, null) genotypes with different clinical and biochemical parameters including sex, serum fasting blood glucose levels, $\mathrm{HbA}_{1 \mathrm{c}}$, lipid profiles and blood pressure in diabetic subjects. Our study showed significant differences in both triglycerides and VLDL-C for GSTT1, which was in agreement with Wang et al. (2006), who suggested that GSTT1-null has an effect on blood lipids. We did not observe any significant effect of GSTT1-null on glycemic control.

Meanwhile, our study showed significant differences in $\mathrm{HbA}_{1 \mathrm{c}}$ and diastolic blood pressure for GSTM1. These results are in agreement with a previous report by Bessa et al. (2009), who concluded that the GSTM1-null genotype may be helpful in identifying 
individuals at high risk for essential hypertension in the Egyptian population. Conversely, Delles et al. (2008) did not find an association between GSTM1 gene variants and hypertension. This discrepancy could be due to differences in ethnic, genetic and environmental background of the population studied. These results also suggested that GSTM1-null has an effect on glycemic control.

In conclusion, our study suggests that the GSTT1 and GSTM1 genes, alone or combined, have an influence on the risk of having type 2 diabetes mellitus. All together, these results suggest that GSTT1 and GSTM1 cooperatively play a protective role against the development of type 2 diabetes mellitus regardless of smoking status. There is an association of GSTM1 (present, null) and GSTT1 (present, null) genotypes with different clinical and biochemical parameters. Only GSTM1-null has an effect on glycemic control.

\section{REFERENCES}

Ada AO, Suzen SH and Iscan M (2004). Polymorphisms of cytochrome P450 1A1, glutathione S-transferases M1 and T1 in a Turkish population. Toxicol. Lett. 151: 311-315.

American Diabetes Association (2007). Diagnosis and classification of diabetes mellitus. Diabetes Care 30 (Suppl 1): 47. Baynes JW (1991). Role of oxidative stress in development of complications in diabetes. Diabetes 40: 405-412.

Bessa SS, Ali EM and Hamdy SM (2009). The role of glutathione S- transferase M1 and T1 gene polymorphisms and oxidative stress-related parameters in Egyptian patients with essential hypertension. Eur. J. Intern. Med. 20: 625-630.

Bid HK, Konwar R, Saxena M, Chaudhari P, et al. (2010). Association of glutathione S-transferase (GSTM1, T1 and P1) gene polymorphisms with type 2 diabetes mellitus in north Indian population. J. Postgrad. Med. 56: 176-181.

D'Alo F, Voso MT, Guidi F, Massini G, et al. (2004). Polymorphisms of CYP1A1 and glutathione S-transferase and susceptibility to adult acute myeloid leukemia. Haematologica 89: 664-670.

Delles C, Padmanabhan S, Lee WK, Miller WH, et al. (2008). Glutathione S-transferase variants and hypertension. $J$. Hypertens. 26: 1343-1352.

Doney AS, Lee S, Leese GP, Morris AD, et al. (2005). Increased cardiovascular morbidity and mortality in type 2 diabetes is associated with the glutathione $\mathrm{S}$ transferase theta-null genotype: a Go-DARTS study. Circulation 111: 2927-2934.

Douglas KT (1987). Mechanism of action of glutathione-dependent enzymes. Adv. Enzymol. Relat. Areas Mol. Biol. 59: 103-167.

Garte S, Gaspari L, Alexandrie AK, Ambrosone C, et al. (2001). Metabolic gene polymorphism frequencies in control populations. Cancer Epidemiol. Biomarkers Prev. 10: 1239-1248.

Gorogawa S, Kajimoto Y, Umayahara Y, Kaneto H, et al. (2002). Probucol preserves pancreatic beta-cell function through reduction of oxidative stress in type 2 diabetes. Diabetes Res. Clin. Pract. 57: 1-10.

Gsur A, Haidinger G, Hinteregger S, Bernhofer G, et al. (2001). Polymorphisms of glutathione-S-transferase genes (GSTP1, GSTM1 and GSTT1) and prostate-cancer risk. Int. J. Cancer 95: 152-155.

Hayes JD and McLellan LI (1999). Glutathione and glutathione-dependent enzymes represent a co-ordinately regulated defence against oxidative stress. Free Radic. Res. 31: 273-300.

Hayes JD and Strange RC (2000). Glutathione S-transferase polymorphisms and their biological consequences. Pharmacology 61: 154-166.

Hori M, Oniki K, Ueda K, Goto S, et al. (2007). Combined glutathione S-transferase T1 and M1 positive genotypes afford protection against type 2 diabetes in Japanese. Pharmacogenomics 8: 1307-1314.

London SJ, Yuan JM, Chung FL, Gao YT, et al. (2000). Isothiocyanates, glutathione S-transferase M1 and T1 polymorphisms, and lung-cancer risk: a prospective study of men in Shanghai, China. Lancet 356: 724-729.

Maritim AC, Sanders RA and Watkins JB, III (2003). Diabetes, oxidative stress, and antioxidants: a review. J. Biochem. Mol. Toxicol. 17: 24-38.

Pemble S, Schroeder KR, Spencer SR, Meyer DJ, et al. (1994). Human glutathione S-transferase theta (GSTT1): cDNA cloning and the characterization of a genetic polymorphism. Biochem. J. 300: 271-276.

Shaikh RS, Amir M, Masood AI, Sohail A, et al. (2010). Frequency distribution of GSTM1 and GSTT1 null allele in Pakistani population and risk of disease incidence. Environ. Toxicol. Pharmacol. 30: 76-79.

Shaw JE, Sicree RA and Zimmet PZ (2010). Global estimates of the prevalence of diabetes for 2010 and 2030. Diabetes Res. Clin. Pract. 87: 4-14. 
Sheehan D, Meade G, Foley VM and Dowd CA (2001). Structure, function and evolution of glutathione transferases: implications for classification of non-mammalian members of an ancient enzyme superfamily. Biochem. J. 360: 1-16.

Stephens JW, Khanolkar MP and Bain SC (2009). The biological relevance and measurement of plasma markers of oxidative stress in diabetes and cardiovascular disease. Atherosclerosis 202: 321-329.

Wang G, Zhang L and Li Q (2006). Genetic polymorphisms of GSTT1, GSTM1, and NQO1 genes and diabetes mellitus risk in Chinese population. Biochem. Biophys. Res. Commun. 341: 310-313.

Wild S, Roglic G, Green A, Sicree R, et al. (2004). Global prevalence of diabetes: estimates for the year 2000 and projections for 2030. Diabetes Care 27: 1047-1053. 